\title{
Control of the inhibiting capability of a spiropyran in anodic dissolution of lead in water-acetonitrile environment by light flux
}

\author{
V. V. Ekilik,* K. S. Tikhomirova and A. G. Berezhnaya
}

Southern Federal University, ul. Zorge 7, Rostov-on-Don, 344090 Russian Federation

*E-mail:v.ekilik@yandex.ru

\begin{abstract}
It has been found that the inhibiting effect of a spiropyran is enhanced under illumination and that this enhancement increases with the spiropyran concentration. The anodic dissolution of lead and cathodic reduction of films in water-acetonitrile media has been studied as a function of electrolyte nature and water content. It has been determined that the passive state of lead starts before the corrosion potential and changes to local depassivation, while water has an activating effect.
\end{abstract}

Key words: photocontrolled inhibitor, spiropyran, lead, water-acetonitrile solutions of tetrabutylammonium and lithium perchlorates, anodic dissolution, depassivation, repassivation.

Received: September 3, 2012.

doi: $\underline{10.17675 / 2305-6894-2012-1-2-080-085}$

\section{Introduction}

It is well known that the effect of organic additives on electrode reactions results from the formation of surface complexes or organometallic compounds. It was found in recent years that complexation in solutions can be controlled by light [1]. This possibility is based on photochromic transitions of molecular structures between two isomeric forms. The shift of equilibrium can also be initiated by metal ions due to stabilization of one of the forms when a complex is formed. Spiropyran derivatives are typical representatives of this class of ligands. The specifics of spiropyrans is that, due to interaction with a metal ion, not only electron density redistribution occurs in the ligand but also the original ligand structure changes. If spiropyrans are used as potential ligands, the coordination-active form can be generated by a light flux. The reverse process, i.e., photoinduced dissociation of the complex, is also possible [2]. It could therefore be expected that spiropyrans would act as photo-controlled regulators of anodic metal dissolution featuring optical response. It seemed worthwhile to study the efficiency of one representative of this class of compounds toward lead.

\section{Experimental}

Electrochemical measurements were carried out by common techniques in a threeelectrode cell with a quartz window for light transmission. Forward polarization curves in 
the direction of potential $(E)$ increase from $-1 \mathrm{~V}$ to $0 \mathrm{~V}$ and reverse curves in the same potential range were recorded using stepwise $E$ variation with a step of $50 \mathrm{mV}$ and exposure for $1 \mathrm{~min}$ at each $E$ value. In addition, anodic chronoamperograms in the passive region at $E=-0.3 \mathrm{~V}$ as well as cathodic chronopotentiograms were recorded. An EVL-1M saturated silver chloride electrode was used as the reference and the $E$ values are reported with respect to it. A lead electrode with a disc-shaped working surface (area $=0.2 \mathrm{~cm}^{2}$ ) was mechanically abraded, polished, and cathodically activated for $15 \mathrm{~min}$ at current density $i_{c}=100 \mathrm{~A} / \mathrm{m}^{2}$ in the working solution with simultaneous sparging with electrolytic hydrogen. The studies were carried out $0.1 \mathrm{M}$ solutions of tetrabutylammonium and lithium perchlorates in acetonitrile containing $0.4 \%$ or $5 \%$ water. The electrode was illuminated in a cell with a quartz window using a $75 \mathrm{~W}$ halogen lamp. 8-Methoxy-1,3',3'trimethylspiro[chromen-2,2-indoline]-6-carbaldehyde synthesis of which is reported elsewhere [3] was used as the additive.

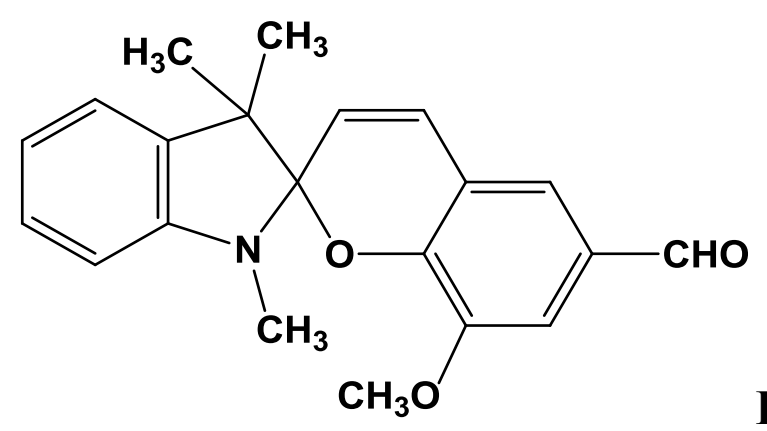

\section{Results and Discussion}

Spiropyrans are nearly insoluble in water, so the studies were carried out in acetonitrile with a suitable electrolyte. Since there are no literature data on the effects of electrolyte composition and water admixtures on the anodic dissolution of lead in these media, the corresponding results precede the data on the use of spiropyran $\mathbf{I}$ as an inhibitor.

The shape of the forward anodic curves is typical of passive metals. Anodic polarization results in lead depassivation (Fig. 1). The anodic current density $i_{a}$ in a tetrabutylammonium perchlorate solution is 2-4 times smaller than that in lithium perchlorate solutions, but the pitting potential $E_{p}$ and repassivation potential $E_{r p}$ on the forward and reverse curves, respectively, are almost independent of the cation nature. The same applies to currentless potentials $E_{i=0}$ on the reverse curves and may suggest a similarity of the compositions of the passive films that most likely consist of $\mathrm{PbO}$ and perhaps a basic salt, and that are formed with involvement of water. The latter is suggested by an inflexion on the anodic curve in a lithium perchlorate solution, as previously observed in aqueous sulfate media [4]. However, the film thickness differs considerably. Two cathodic peaks on the reverse polarization curve in a lithium perchlorate solution are observed, whereas the peaks are nearly nonexistent in a tetrabutylammonium perchlorate solution. Accordingly, in this solution there are no potential arrests on the cathodic 
chronopotentiogram recorded after the anodic chronoamperogram in the passive region. Hence, it can be believed that adsorption of the tetrabutylammonium cation prevents the film formation. The forward and reverse cathodic polarization curves at negative potentials nearly coincide, suggesting that the film reduction is sufficiently complete.

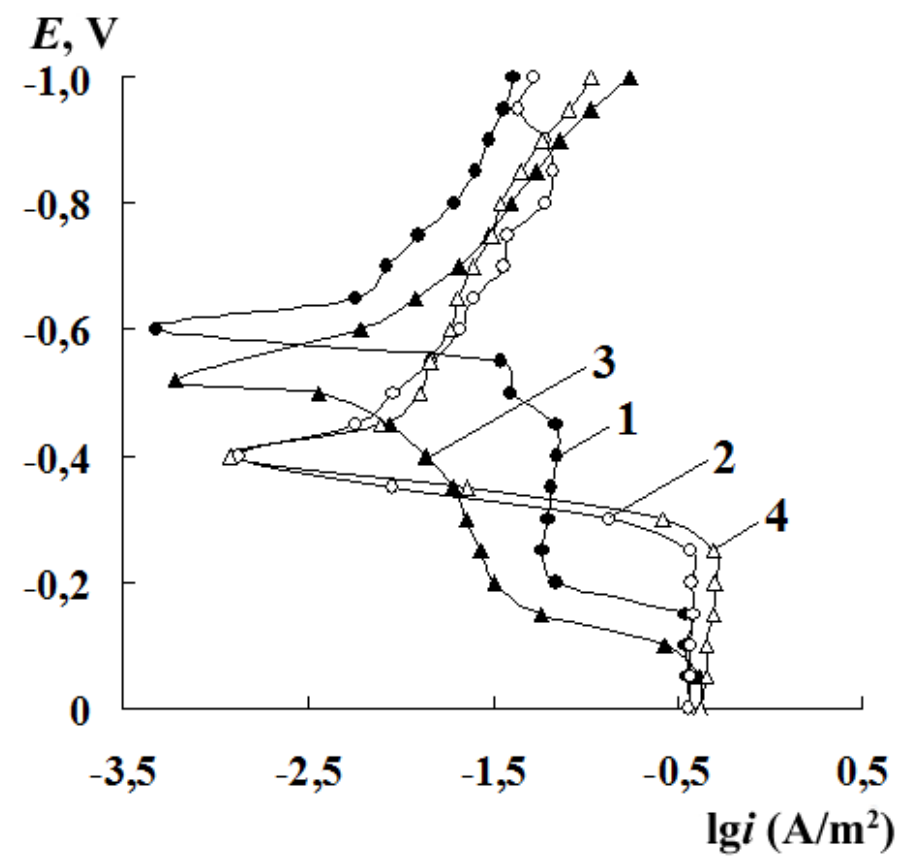

Figure 1. Forward $(1,3)$ and reverse $(2,4)$ polarization curves in $0.1 \mathrm{M}$ solutions of lithium perchlorate $(1,2)$ and tetrabutylammonium perchlorate $(3,4)$.

An increase in the water concentration $\left(C_{\mathrm{H}_{2} \mathrm{O}}\right)$ to $5 \%$ in the tetrabutylammonium perchlorate solution considerably decreases the corrosion potentials $E_{c o r}, E_{p}$, and $E_{r p}$ and increases $i_{a}$ by an order or more (Fig. 2). Regions of kinetic and mixed control appear on the anodic chronoamperogram (Fig. 3). The current is stable until $\tau=20 \mathrm{~s}$. In the range $\tau=$ $20 \ldots 120 \mathrm{~s}$, the plot of $i_{a} v s . \tau$ is described by a straight line equation $i_{a}^{-1}=0.6+0.02 \cdot \tau^{-1 / 2}$ with correlation coefficient $R=0.99$ that is typical of mixed control [5]. It is only at $\tau>240 \mathrm{~s}$ that the straight line equation $i_{a}=15.5 \cdot \tau^{-1 / 2}$ with $R=0.99$ is realized, which suggests that non-steady-state diffusion at constant diffusion coefficient is the slow stage. Conversely, a similar curve $i_{a}=0.62 \cdot \tau^{-1 / 2}$ with $R=0.97$ describes the entire range studied in the solution with $C_{\mathrm{H}_{2} \mathrm{O}}=0.4 \%$. The slope of Cottrell's straight line is 25 times higher, which suggests that the film permeability is higher at $C_{\mathrm{H}_{2} \mathrm{O}}$. An increase in $C_{\mathrm{H}_{2} \mathrm{O}}$ brings about two potential arrests on the cathodic chronopotentiogram recorded after the anodic chronoamperogram (Fig. 3) and two peaks on the reverse cathodic polarization curve (Fig. 2). In this case, the second potential arrest and the peak, which most likely 
correspond to $\mathrm{PbO}$ reduction, are larger. Thus, water activates dissolution and considerably increases the film thickness.

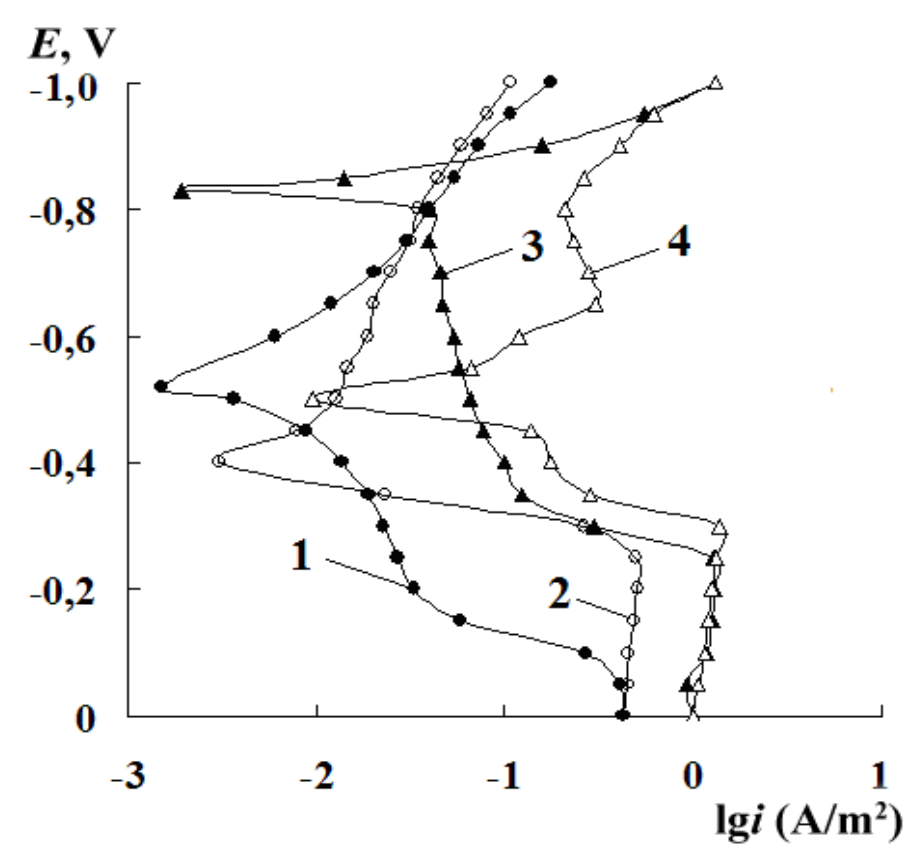

Figure 2. Forward $(1,3)$ and reverse $(2,4)$ polarization curves in $0.1 \mathrm{M}$ solutions of tetrabutylammonium perchlorate at $C_{\mathrm{H}_{2} \mathrm{O}}=0.4 \%(1,2)$ and $5 \%(3,4)$.

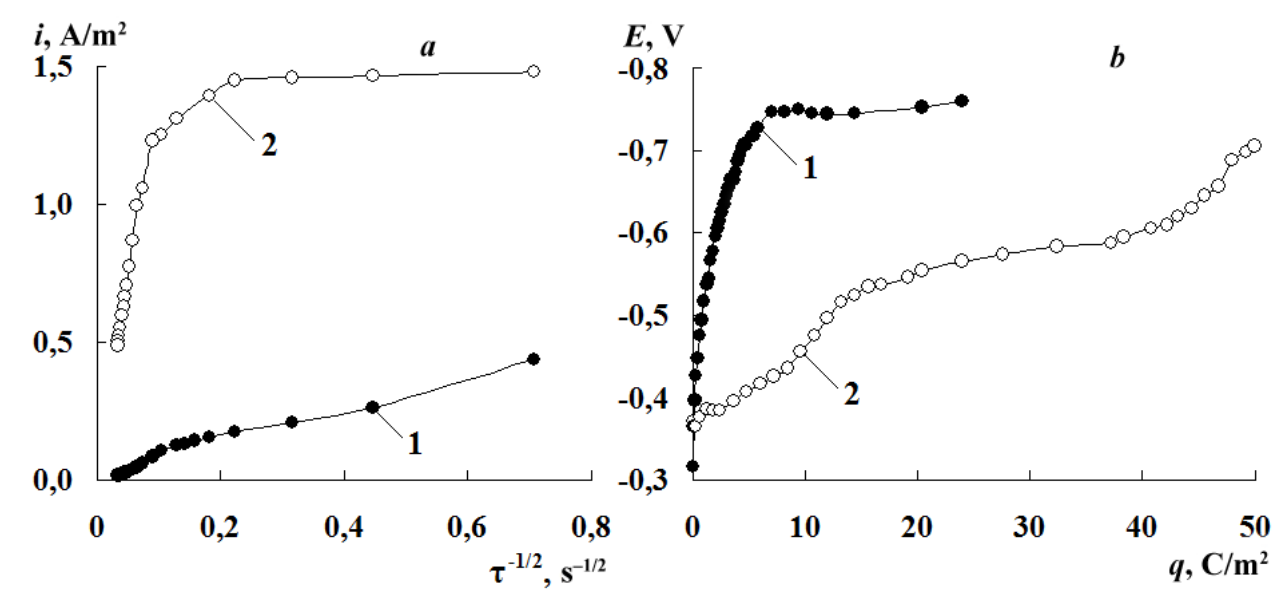

Figure 3. Anodic chronoamperograms at $E=-0.3 \mathrm{~V}(a)$ and cathodic chronopotentiograms (b) in $0.1 \mathrm{M}$ solutions of tetrabutylammonium perchlorate without an additive at $C_{\mathrm{H}_{2} \mathrm{O}}=0.4 \%$ (1) and $5 \%(2)$.

Spiropyran I affects the lead dissolution rate in the passive region and upon depassivation but nearly does not affect $E_{p}$ (Fig. 4). Though chemical synthesis of a complex from a metal cation and a spiropyran usually does not occur without illumination, the additive still has some efficiency. This is apparently due to specifics of chemisorption, 
as indicated, e.g. in [6], electrochemical synthesis of complexes under conditions where chemical synthesis is impossible.

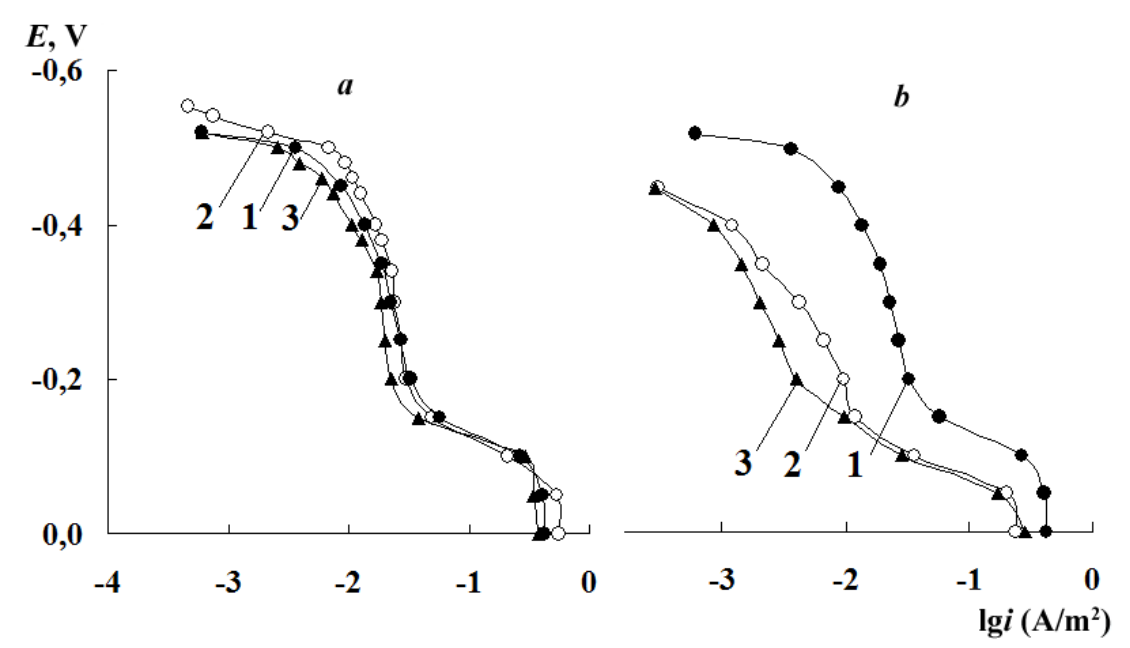

Figure 4. Forward polarization curves in $0.1 \mathrm{M}$ tetrabutulammonium perchlorate solutions with $C_{\mathrm{H}_{2} \mathrm{O}}=0.4 \%$ without an additive ( 1 ) and in the presence of spiropyran $\mathbf{I}$ at $C=0.1 \mathrm{mmol} / \mathrm{l}(a)$ and $C=0.5 \mathrm{mmol} / \mathrm{l}(b)$, without (2) and with electrode illumination (3).

The inhibiting effect of spiropyran I increases with its concentration and under illumination. At $C=0.1 \mathrm{mmol} / \mathrm{l}$, the insignificant stimulating effect of the additive with an increase in potential changes to weak inhibition (no more than 10\%). Illumination decreases the dissolution rate by $30-40 \%$ in a solution containing spiropyran $\mathbf{I}$, so the latter becomes an inhibitor at all potentials. At $C=0.5 \mathrm{mmol} / \mathrm{l}$, the additive behaves as an inhibitor in all cases, although its effect decreases somewhat with an increase in potential. Without illumination, it decreases the dissolution rate 3-9 fold depending on potential, in comparison with a 8-14 fold decrease with illumination. Small pits are clearly visible on the electrode surface after recording polarization curves and chronoamperograms. However, they are nearly invisible if the curves are recorded in the presence of the additive, especially under illumination.

We are grateful to A. V. Chernyshev and N. A. Voloshin for the compounds they provided.

\section{Conclusion}

Spiropyran I hinders dissolution at all potentials but does not affect the pitting potential. Illumination of the electrode surface enhances the inhibiting effect of the ionochromic additive. Water accelerates the process on lead in acetonitrile solutions of tetrabutylammonium perchlorate both in the passive state and in the case of local depassivation by facilitating the formation of a thicker film. A similar effect is observed on transition to lithium perchlorate solutions. 


\section{References}

1. M. Inouye, Coord. Chem. Rev., 1996, 148, 265.

2. A. K. Chibisov and H. Görner, J. Chem. Soc., Faraday Trans., 1998, 94, 2557.

3. A. V. Chernyshev, M. S. Chernov'yants, E. N. Voloshina and N. A. Voloshin, Russ. J. Gen. Chem., 2002, 72, no. 9, 1468.

4. V. V. Ekilik, K. S. Tikhomirova and A. G. Berezhnaya, Fiz. Khim. Poverkhn. Zashch. Met., 2011, 47, no. 3, 302.

5. V. V. Ekilik, A. G. Berezhnaya and V. A. Tugolukova, Korroz.: Mater. Zashch., 2004, no. 1, 16 (in Russian).

6. N. N. Bogdashev, Extended Abstract of Cand. Sci. Dissertation, Rostov-on-Don, RGU, 1974 (in Russian). 